\title{
The other side of breaking bad news
}

\author{
Cite as: CMAJ 2020 November 23;192:E1548-9. doi: 10.1503/cmaj.201663
}

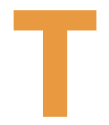

here is an old saying that a person with their health has 1000 dreams, but a person without it only has one.

My husband and I met eight years ago in Key West, Florida. He lived in the United States and I, in Canada. Many flights, FaceTime calls, text messages, emails, phone calls and a wedding later, in July 2019 we were finally both living in the same city and officially beginning our life together. Then, in November 2019, our lives changed forever.

It happened early on a Friday morning. Just a few weeks prior, we had run a half-marathon together. At 4 am, Brian woke me up because he had a bad leg cramp. I saw his leg jerking under the covers, which soon involved his arm, face and, before I knew it, he lost consciousness, stopped breathing and became blue. This is how our nightmare began. On the ambulance ride, I thought about causes of new onset seizures in adults. I feared the worse.

After Brian's computed tomography (CT) scan, the emergency physician updated us before the radiology report was available. To his eyes, the scan looked normal. I could breathe again. But that was short lived. A few moments later, the official radiology report came up and we learned, in the hallway of a busy tertiary centre, that there was in fact a lesion, most likely "neoplastic." The look of confusion on my husband's face let me know that I needed to compose myself to explain what neoplastic meant. My nonmedical husband did not understand his diagnosis.

What followed over the next few months was more bad news. We saw neurosurgery only to learn that the tumour was inoperable because of its location in the brain. But that was fine, we were told, because the magnetic res-

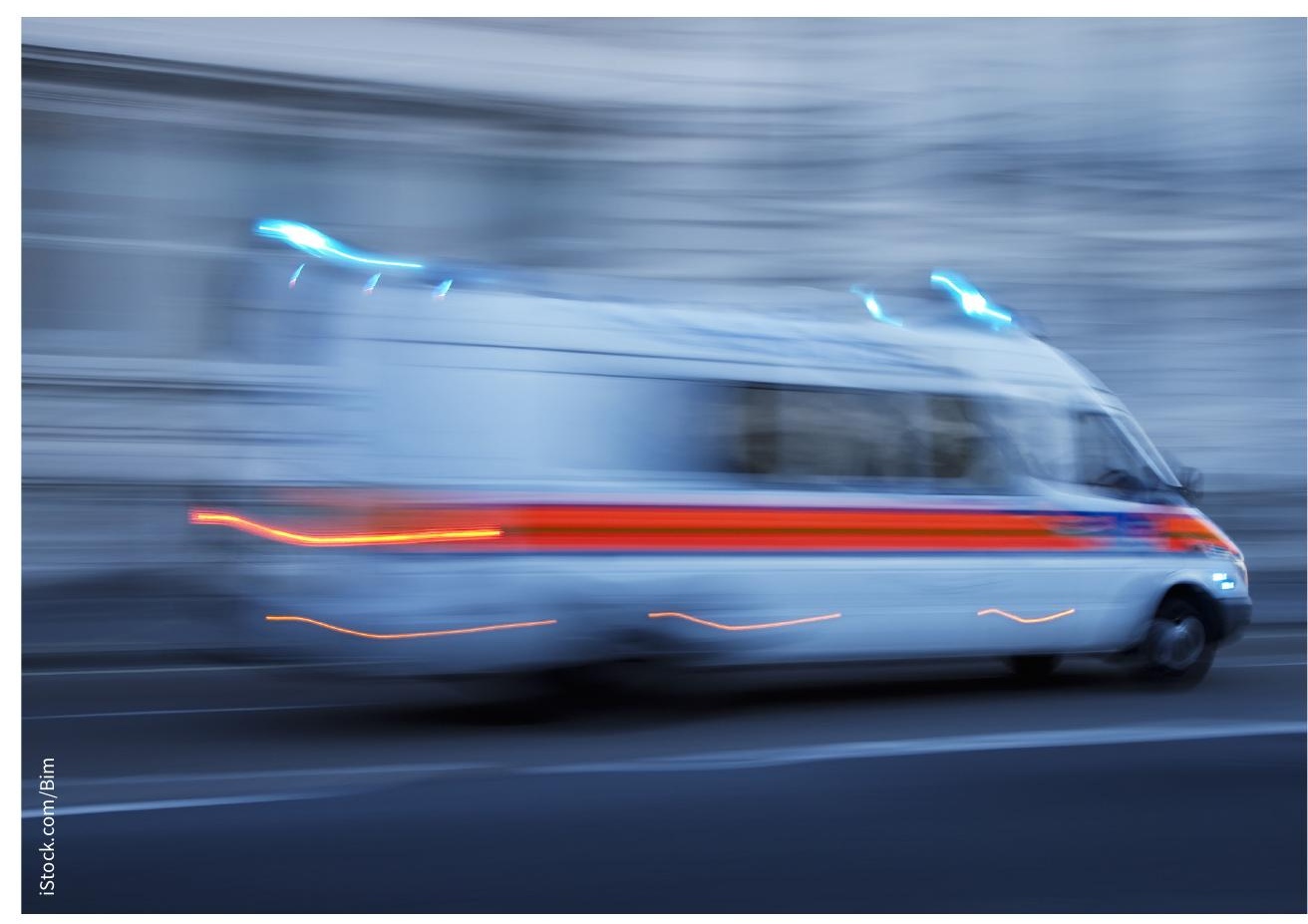

onance image (MRI) characteristics showed that this was a low-grade glioma. We had years, possibly decades before any decisions needed to be made. We met with neurology, who prescribed antiepileptic drugs to keep Brian's seizures under control. We decided to monitor the tumour with another scan in two months.

More bad news followed. The tumour had grown, making it a higher-grade glioma. But that too, was fine because, given that Brian was young, it likely had a mutation in a gene, the IDHI gene. It was therefore more likely to respond to chemotherapy and radiation. The prognosis was good. But, still, life was going to change because Brian had brain cancer, our oncologist said. To this day, this does not sound real.

After the biopsy, we learned that the preliminary pathology report said the tumour was either a grade 3 anaplastic astrocytoma or glioblastoma, and it did not have any mutations to make it more susceptible to treatment. This meant a worse prognosis than anticipated. No one ever reviewed the final pathology and its implications with us. It was up to me to check the results on the patient portal and tell Brian his final diagnosis. This was unfortunate because, although the treatment plan was not affected by the final pathology, the prognosis was different.

Now, a few months out from that diagnosis, Brian has completed concurrent radiation and chemotherapy, and will remain on chemotherapy for one year. I took time away from my fellowship to help him during treatment. My colleagues and my fellowship program have been tremendously supportive. My program director was one of the first people I reached out to on diagnosis day. He helped me navigate the situation and ensured that taking time off and then returning to work was seamless. Other staff contacted Brian and I 
weekly. My mentor continues to be a tremendous force in getting my slightly derailed career plans back on track. It is hard to describe how much that means to me and to Brian. Receiving a diagnosis of brain tumour is a very lonely and alienating experience, but the support that we've received from colleagues, friends and family has been unbelievable and made it somewhat bearable.

I have learned so much over these last few months and not only about brain tumours. Our journey made me reflect on being a patient, a caregiver and a physician. Some of our experiences have been positive - we have had physicians make themselves available to us outside of clinic times, and some have gone above and beyond to help us process the diagnosis and manage Brian's symptoms, and some have pointed us in the direction of second, third and fourth opinions.

The other experiences I am using as learning opportunities. In medical school, we are taught how to break bad news. Having been on the receiving end of badly delivered bad news, I cannot emphasize that enough. Physicians should be mindful of their "setting" and bad news should be delivered in a private environment, not in a hallway. Perhaps it was the time and space constraints that led that emergency physician to tell us about the CT findings in the hallway, but Brian will always remember getting his diagnosis in a busy emergency department environment without privacy. I still find it difficult to assess patients in that emergency department because of the memories it brings back.

I believe professionalism and empathy are core components of being a physician. We are taught to empathize with the patient's emotion. I would encourage all caregivers to recognize a patient's emotions and never dilute their experience or attribute their diagnosis to bad luck. Patients will always remember how they were told their lives will change forever. Physicians should tailor the information to the level of the understanding of the patient not their partner, even if the partner is a physician.

In medical school, we are also taught to deliver bad news in manageable morsels of information. That is what happened to us, in a manner of speaking, over the span of a few months. It felt as though every time we managed to compose ourselves after receiving bad news, we would get floored again by even worse news and that was emotionally and psychologically draining for both of us. It made me wonder if perhaps receiving all the bad news at once would have been better, breaking us only once, not allowing us false hope only to tear us down several times over.

During Brian's treatment, I struggled with being a physician and caregiver and with identifying what was expected of me. At times, I was a caregiver who was expected to accept direction and forget my medical training. At my most vulnerable, when my personal life was coming apart and my role should have been that of spouse, I was expected to be a medical professional and deliver information about the diagnosis to my husband, even though I had no in-depth knowledge about brain cancer. It was practically impossible for me to provide this information. At times I was comfortable with the role of advocate and physician, but other times I wished the medical team would recognize that oncology was not my area of expertise and provide the guidance that I did not feel we were receiving. But sometime when I was advocating for Brian and making perfectly sound medical requests, such as obtaining an electrocardiogram before starting a QT-prolonging medication, I was met with doubt by Brian's physicians.

Today, I interact with patients differently. I take more time to explain their diagnosis, choose my words more carefully, and offer treatment and contingency plans. The most difficult experience I had to come to terms with was the sense of powerlessness. As a caregiver, this feeling suffocated me in a way I had never understood as a physician. Now, to mitigate the lack of control that comes with certain diagnoses, I offer patients information on things they can control, such as diet or integrative medicine (provided it does not interfere with conventional therapy). Those things gave me control when I needed it most.

People without their health have only one dream: to get it back. The diagnosis crushed us, but our world did not stop. Since then we have been working on getting Brian's health back. We have grown closer, we started making healthier food choices, we are more mindful. As for what the future holds, who knows? Brian is a positive person and I am thankful for that. There is no denying that we have dark days as well, but we are choosing not to focus on statistics. We are living in the present and maintaining as normal a life as possible, working toward the same personal, family and professional goals that we had before diagnosis day, perhaps with a slightly enhanced sense of urgency.

\section{Roxana Chis MD}

Division of Gastroenterology and Hepatology, Department of Medicine, University of Toronto, Toronto, Ont.

This article has been peer reviewed.

This is a true story. Brian has given his consent for this story to be told. 